\title{
Marseille : réseaux migrants transfrontaliers, place marchande et économie de bazar
}

\section{Michel Peraldi}

\section{(2) OpenEdition \\ 12 Journals}

Édition électronique

URL : http://journals.openedition.org/conflits/232

DOI : $10.4000 /$ conflits.232

ISSN : $1777-5345$

Éditeur :

CCLS - Centre d'études sur les conflits lilberté et sécurité, L'Harmattan

Édition imprimée

Date de publication : 15 mai 1999

ISSN : 1157-996X

Référence électronique

Michel Peraldi, « Marseille : réseaux migrants transfrontaliers, place marchande et économie de bazar », Cultures \& Conflits [En ligne], 33-34 | printemps-été 1999, mis en ligne le 16 mars 2006, consulté le 30 mars 2021. URL : http://journals.openedition.org/conflits/232 ; DOI : https://doi.org/ $10.4000 /$ conflits. 232

Ce document a été généré automatiquement le 30 mars 2021.

Creative Commons License 


\title{
Marseille : réseaux migrants transfrontaliers, place marchande et économie de bazar
}

\author{
Michel Peraldi
}

1 La plupart des grands programmes d'étude réalisés ces quinze dernières années sur l'intégration des migrants aux marchés du travail dans les pays occidentaux signalent une mutation assez radicale des processus d'affectation et des destinations professionnelles des populations migrantes. En effet, sociologues, géographes et anthropologues, semblent d'accords pour souligner le développement de la petite entreprise artisanale ou commerciale dans ces mêmes mondes migrants qui, voici peu encore, étaient pour l'essentiel destinés à occuper les moins qualifiés des emplois ouvriers. Cette réaffectation est loin sans doute d'absorber et de compenser l'importance des mises à pied et tout particulièrement le chômage massif de la seconde génération issue de l'immigration. Elle est néanmoins suffisamment significative pour ne plus être considérée comme un épiphénomène singulier et statistiquement invisible, assimilable à la permanence de formes économiquement archaïques. Une étude statistique menée récemment en France signale par exemple que 17\% des migrants récents originaires du Maghreb sont artisans, commerçants ou chefs d'entreprise ${ }^{1}$. Cette observation confirme des tendances déjà significatives depuis les années 70 dans la plupart des pays occidentaux ${ }^{2}$. Aux Etats-Unis plus encore, ce mouvement d'intégration des migrants vers la petite entreprise est connu dans son ampleur et son dynamisme ${ }^{3}$ et il semble désormais évident qu'un migrant, issu des pays asiatiques ou d'Amérique Latine a aujourd'hui, aux Etats Unis, plus de chance de trouver un ancrage professionnel dans une «niche économique ethnique » que dans le marché ouvert du travail.

2 Cette réorientation est à comprendre bien sûr dans le mouvement général des économies occidentales, comme une pièce des redéploiements des marchés du travail : le transfert des mains-d'œuvre ouvrières fordistes vers le secteur tertiaire, le développement de la petite production et des chaînes de sous-traitance, la 
précarisation et la fluidité qui favorise le recours ponctuel à des mains-d'œuvre instables. L'entrepreneur migrant doit être compris comme l'une des figures émergentes de cette fin de siècle, à côté de toutes les nouvelles figures laborieuses qu'engendrent la précarité, la flexibilité et la mondialisation. Reste qu'elle est probablement l'une des plus énigmatiques. En effet, on connaît bien le fonctionnement de «l'ethnic business » dès l'instant où ce type d'entreprise occupe une place spécifique dans les économies urbaines, indexée sur l'existence d'enclaves résidentielles à consistance ethnique. Il a également été assez clairement montré les logiques de contrainte qui gouvernent ce type d'accès au travail et ses modes spécifiques de gestion de la précarité ${ }^{4}$. Mais ces deux orientations de recherche semblent insuffisantes dès lors que l'entrepreneuriat migrant fait dispositif dans la ville et peut prétendre à organiser non pas seulement des "créneaux " économiques particuliers intégrés à l'existence de communautés structurées, mais des pans entiers de l'économie urbaine, licite et illicite, et notamment dans son interface local-global. Car les paradigmes explicatifs qui relèvent, soit de "l'économie ethnique », soit de la logique de survie semblent un peu courts dès l'instant où l'on est confronté à des dispositifs qui constituent des "chaînes entrepreneuriales » impliquant des populations ethniquement diversifiées d'une part, et d'autre part combinant autour de leurs activités des formes de sédentarité et de nomadisme. Ces quinze dernières années sont en effet apparues des formes entrepreneuriales très différentes de celles décrites usuellement comme représentatives de l'économie ethnique: soit le commerce des biens communautaires (épicerie, services), soit la reconversion en entreprises artisanales des métiers que les migrants occupaient jusqu'alors au titre d'ouvriers (dans le secteur du bâtiment et des travaux publics essentiellement). On voit en effet se développer aujourd'hui des réseaux d'entreprises et d'acteurs migrants, désormais capables d'organiser des circulations de marchandises et des «filières » marchandes à l'échelle internationale, entre les pays originaires et les pays de migration, dans un sens ou l'autre selon la nature du produit; en somme, non pas des entreprises culturellement solidaires et économiquement concurrentes, mais des "chaînes entrepreneuriales » combinant les activités séculaires du négoce et du colportage à rayon international.

3 Le cas emblématique de Marseille

4 Dans le quartier Belsunce, au cœur du « centre historique » de la ville, périmètre borné d'un côté par la gare de chemin de fer, de l'autre par le port de commerce, s'est établi, sur la base de commerces ethniques de proximité installés en ces lieux depuis le début $\mathrm{du}$ siècle, un dispositif commercial constituant l'un des principaux lieux d'approvisionnement pour les communautés maghrébines, et depuis peu africaines, vivant de part et d'autre de la Méditerranée. Dans les années 80 à 88 , quelques 400 boutiques y réalisaient alors un chiffre d'affaires estimé à trois milliards et demi de francs grâce à un flot de chalandise de plus de 40000 personnes venant du Maghreb les week-end, auquel s'ajoutaient des consommateurs locaux et régionaux ${ }^{5}$. Ce dispositif rassemble non seulement tous les produits vivriers introuvables dans les commerces européens, notamment la viande hàlal, les épices, et autres produits spécifiques, mais également tous les produits manufacturiers difficiles d'accès dans les pays d'origine : tissus, pièces automobiles, électroménager, voitures neuves ou d'occasion, etc. La façade commerciale a été ouverte par des commerçants algériens, arrivés à Marseille dans les années 70-75, auxquels se sont ajoutés depuis des commerçants tunisiens, sénégalais, marocains. Mais plus fondamentalement, ce dispositif s'appuie sur d'amples 
réseaux commerciaux, pour partie installés sur le quartier, comme les grossistes en confection, juifs séfarades, originaires du Maroc et d'Algérie, qui se sont établis à Marseille après la décolonisation, pour partie sur des fournisseurs et grossistes dispersés entre la Belgique pour les tapis, l'Allemagne pour les voitures, l'Italie pour le cuir et la chaussure, le transit de l'électroménager asiatique, la Turquie pour les bijoux, la confection. Ce « comptoir colonial ${ }^{6}$ n'est donc pas qu'une surface commerciale. Il est surtout un lieu de rupture de charges sur des routes commerciales qui articulent le Nord productif et le Sud consommateur et sur lesquelles circulent des marchandises et des hommes, des capitaux et des informations. La spécificité de ce dispositif commercial tient essentiellement au fait que les circulations sont assurées en grande partie hors de toute logistique normée de transport, soit par les commerçants euxmêmes, soit par des réseaux de "fourmis » et colporteurs. Le quartier Belsunce est certes un lieu de commerces de proximité et d'échanges marchands directs. Mais c'est aussi une place de négoce qui crée les conditions économiques et sociales d'échanges à l'échelle circum-méditerranéenne pour tous les produits soumis à des interdits sociaux ou à des embargos politiques: l'or déréglementé qui arrive d'Istanbul ou de Naples pour transiter vers le Maghreb et l'Afrique ; les voitures allemandes qui transitent vers la Libye lorsque ce dernier pays est soumis à embargo, mais également les baskets américains à prix coûtant, les fins de séries et les invendus, les imitations ou les contrefaçons de produits de luxe, etc. Ces quelques exemples pour dire que Belsunce est d'abord une place marchande où, par l'initiative des acteurs, sur fond d'accords oraux et d'engagements réciproques, des marchandises reprennent leur circulation et leur cycle de profitabilité interrompus par les filtres sociaux et politiques, passent les différentiels de richesse. Fluctuant au gré des relations politiques entre l'Europe et les pays du Maghreb, souvent fragilisé par les crises politiques qui secouent l'Algérie notamment, soumis à la pression urbanistique des institutions locales et au procès en diabolisation entretenu de façon chronique par l'opinion publique locale, mais également sans cesse dynamisé par les initiatives entrepreneuriales qu'il localise, le comptoir commercial ne cesse d'évoluer. C'est en effet dans un rapport de succession/ coopération et un jeu de "chaises musicales " ${ }^{7}$ que s'inscrivent les différentes vagues migratoires qui ont trouvé, dans ce quartier central, tout à la fois un lieu d'installation et un centre d'affaires et d'échanges. Les modes de coopération qui s'instituent entre les " anciens » et les "nouveaux" arrivants permettent un renouvellement constant des zones d'approvisionnement, des routes commerciales, l'assurance d'une maind'œuvre disponible. Les commerçants algériens des années 70-80 ont ainsi succédé aux artisans arméniens et italiens qui occupaient le quartier dans les années 30 à 50, tout en assurant de nouveaux débouchés aux marchandises produites par ceux de ces artisans restés dans le quartier. Ces mêmes Algériens ont été introduits et "parrainés » dans leur installation par les grossistes juifs séfarades, arrivés du Maghreb dès la fin des années 50, à qui ils ont permis de rouvrir les routes commerciales vers le Moyen Orient un temps fermées par la décolonisation. Ce sont ces mêmes Algériens qui, à la fin des années 80 , ont introduit sur le marché, des commerçants africains, sénégalais mourides 8 et des Tunisiens, moyennant là encore de nouveaux accords commerciaux. Perpétuellement évolutif, le dispositif commercial de Belsunce concrétise un agencement interculturel des fonctions commerciales, où, davantage que des relations ethniques, ce sont les flux migrants eux-mêmes et leur différentialités qui servent de ressources mobilisables économiquement. 
5 Le trait le plus significatif de cette évolution est l'ouverture de nouveaux marchés et l'investissement de nouveaux espaces dans la ville, certains à l'immédiate périphérie de ce foyer historique qu'est Belsunce, d'autres, c'est le cas notamment du marché aux Puces ${ }^{9}$, gagnés sur des friches usinaires au cœur des anciens sites industriels des quartiers nord. Ces nouveaux espaces commerciaux, souvent ouverts à l'initiative de commerçants prospères issus du comptoir initial, marquent une nette inflexion des stratégies commerciales : sans perdre forcément leur accrochage direct aux chalandises issues des pays du Maghreb, l'étendant même à l'Afrique, ces nouveaux marchés sont plus systématiquement orientés vers des consommateurs locaux, proposant notamment des produits alimentaires, de la confection, des produits ménagers à très bas prix, calquant en cela leur stratégie sur le modèle des " hard discounters", dont certaines enseignes sont désormais présentes sur ces marchés. Les commerces ainsi redéployés se sont alors plus largement ouverts aux populations locales, celles des mondes de la précarité, mais aussi ces franges des classes moyennes peu contaminées par les clivages culturels et le marquage symbolique d'une distance sociale aux couches populaires. Sur ce nouveau créneau commercial, de nouvelles figures d'entrepreneurs sont apparues, notamment des fils de migrants, jeunes ou moins jeunes chômeurs, exclus du marché du travail salarié, qui tentent là de nouvelles carrières sociales et professionnelles en mobilisant des compétences relationnelles de "passeurs» ou de médiateurs entre les différents univers culturels qui se côtoient sur les marchés ${ }^{10}$. Réseaux migrants: continuités culturelles et discontinuités politiques Le dispositif marseillais ne constitue donc pas une "niche» commerciale ethnique au sens usuellement donné à ce terme en anthropologie urbaine : un lieu de concentration de commerces principalement destinés aux consommations d'une minorité culturelle ou ethnique localement sédentarisée, dans lequel les entrepreneurs appartenant à cette minorité trouvent des conditions économiques et sociales favorables pour échapper à la discrimination dont ils font l'objet sur les marchés du travail et dans les autres zones urbaines. Le commerce algérien occupe certes une place stratégique dans ce dispositif, tant parce que l'Algérie reste un destinataire important des marchandises qui circulent et s'échangent à Marseille ${ }^{11}$ que par la place qu'y occupent les commerçants, sédentaires et mobiles, algériens. Mais, si important soit-il, le commerce algérien n'est ici maintenant qu'une pièce d'un dispositif commercial qui se laisse mal décrire comme enclave minoritaire dans la ville. Toute l'énigme de cette activité commerciale tient justement dans la capacité, singulièrement urbaine, qu'elle manifeste à se diffuser, au niveau local et global, sans perdre les traits constitutifs des formes marchandes et sociales qui l'organisent: l'oralité, la confiance, la fluidité des rôles, soit ce qui globalement en fait une économie du face à face. La co-présence en ces lieux urbains de différentes et successives souches migratoires sédentarisées mais jamais totalement absorbées, dans leur singularité culturelle, par les différents mondes sociaux de la ville ; le maintien de continuités relationnelles aux pays d'origine qui assurent des circulations fluides et un niveau constamment ajusté d'informations sur les situations réciproques des "communautés", et donc la demande, de part et d'autre des différentiels et des frontières; l'essaimage constant d'aventures personnelles dans l'espace organisé par les routes commerciales, tels sont les ingrédients qui assurent la reproduction d'une matrice sociale et économique qui est au fondement du dispositif commercial urbain. Le « réseau » commercial algérien, pour nommer ainsi le dispositif économique exhaustif qui nourrit l'Algérie socialiste en crise, dont Marseille est une pièce et une place marchande essentielle, s'étend aujourd'hui de part et d'autre de la 
Méditerranée. Des voitures allemandes aux tapis belges, de l'électroménager italien à la confection syrienne et turque, le rassemblement, l'échange et le transfert des marchandises fondent l'établissement de commerçants algériens à Istanbul ${ }^{12}$, Damas, Alep, Oujda ou Nador, et bien sûr Marseille ou Paris, bientôt concurrencées par Dubaï et Milan. Voilà ce qu'en substance on peut apprendre, par les nouvelles qui s'échangent sur les uns et les autres, les provenances et les destinations des mouvements et des transits dans les rues de Belsunce. En outre, se dispersant, le commerce laisse, de place marchande en place marchande, des créneaux d'initiative pour d'autres, Tunisiens, Marocains, Libanais, Syriens, Africains. Et chacun de ces nouveaux arrivants, successeurs et concurrents à la fois des "établis ", est une pièce d'autres dispositifs commerciaux de plus ou moins grande amplitude, qui sont à leur tour, chacun à leur manière, en prise sur un marché et des échanges locaux et des dispositifs commerciaux "globaux » : le dispositif algérien croise le dispositif russe sur Istanbul, Alep ou Dubaï, tuniso-lybien ou marocain, africain sur Marseille ou Dubaï. On connaît assez bien maintenant l'ampleur et l'extension du dispositif commercial mouride, le rôle stratégique des "passeurs » tunisiens dans l'approvisionnement de la Libye boycottée par les puissances européennes ${ }^{13}$ mais d'autres croisements et d'autres combinatoires restent à explorer. Toute cette armature d'activités, cette trame "circulatoire", constituent bien un enchevêtrement de réseaux sociaux, pourvu que l'on donne à ce terme d'usage très extensif, son sens anthropologique; c'est à dire une mobilisation ponctuelle, fonctionnelle, de liens sociaux dispersés et constitués dans la dispersion. Bref, non pas des « liens forts » de dépendance à des ordres collectifs et normatifs mais des "liens faibles" ${ }^{14}$ constitués dans le pragmatisme et le détachement qui visent d'abord à réduire les risques et l'incertitude. Les relations commerciales sont sans doute des relations engageantes, organisant des jeux de dettes réciproques et des lacis de dépendances et contre-indépendances individuelles. Mais elles ne semblent jamais assez puissantes pour que des communautés de circonstance se sentent investies d'en porter la responsabilité et d'en garantir l'application des «contrats ». De même, si les désignations ethniques et identitaires imbibent le vocabulaire par lequel les acteurs parlent les uns des autres, reconnaissent des compétences et des spécialités, localisent professionnellement les partenaires ou les concurrents, cette trame langagière ne va pas jusqu'à renvoyer à un partage étanche du marché et à ce qui aurait les apparences d'une division du travail. Celle-ci repose plutôt sur une grammaire individualisée et individualisante du montage des "affaires" et de la position de chacun dans un « milieu » en permanente recomposition, rendu tel par la permanente mobilité spatiale et professionnelle des acteurs eux-mêmes. C'est en effet qu'il s'agit ici d'articuler un rayon commercial local, pour une clientèle dont les goûts et les besoins expriment bien la dispersion sociale qui caractérise les anciennes migrations (des produits culturels " traditionnels » aux modes occidentales pour décliner très sommairement l'inventaire des produits offerts sur les marchés) et un marché lointain, en regroupant des marchandises sur un rayon de fournisseurs qui est à dimension "globale». Ajoutons à la complexité d'une telle activité le fait, décisif pour la compréhension du dispositif, que les marchandises acheminées ne sont pas simplement difficiles à mettre en circulation, pour des raisons techniques qui tiendraient à leur éloignement, leur conservation, etc., mais grosso modo impossibles aux conditions sociales et économiques qui sont celles « artisanales » du dispositif. Tous les acteurs en affaire ici sont installés sur le formidable potentiel d'activités économiques que constitue le paradoxe d'une grande fluidité offerte par la «demande» (pour dire très vite un 
régime de besoins non couverts localement, un appétit consommatoire et les normativités culturelles qui leur assigne tel ou tel produit) et au contraire l'extrême rigidité des régimes de circulation des biens et des marchandises. Soit ce qui constitue en sa forme culturelle, l'opposition paradoxale entre une grande familiarité à des biens, des services, proprement délocalisés, et leur faible accessibilité. Ou encore, pour le dire en qualifiant la forme géopolitique qui sous-tend ce marché, le grand contraste entre la plasticité diasporique des mondes migrants et la rigidité des territorialités politiques et sociales que ces continuités diasporiques transversalisent.

Pour des Algériens vivant en Algérie il est assez commun de se représenter comme légitime, rationnel, désirable et souhaitable, bref, socialement possible, de venir en France (et pour d'autres au Maroc, en Tunisie, en Syrie ou en Turquie) se soigner, s'éduquer, s'habiller ou s'équiper sans nécessairement avoir à émigrer, simplement parce qu'ils sont assurés de trouver dans le réseau diasporique de leurs semblables dispersés par les vagues migratoires anciennes, les raisons et les occasions de cette représentation. Le dynamisme des places marchandes repose sur ce fait culturel (et ce que nous avançons des Algériens peut bien sûr s'appliquer à d'autres peuples migrants). Les dispositifs commerciaux dont nous parlons ici ne sont donc que la partie visible et métropolitaine de la formidable trame de liens, d'informations, de circulations continues par lesquels les mondes migrants maintiennent des adhérences et des continuités aux mondes "originaires", d'autant plus vivaces que les migrations sont anciennes et le courant continu, quelles que soient par ailleurs les distances sociales et les régimes d'écarts qui s'opèrent entre les deux mondes.

L'économie de bazar

8 Il est interdit d'importer en Algérie des voitures de plus de trois ans. Depuis 1997, l'Etat algérien interdit même l'importation de véhicules usagés non accompagnés, et bien sûr, depuis longtemps, le dinar n'est convertible dans aucune des monnaies à rayonnement international, tandis que les ressortissants algériens sont soumis, comme la plupart des étrangers "non-européens ", à des conditions d'obtention de visas de plus en plus draconiennes. A ces difficultés spécifiques s'ajoutent les difficultés inhérentes aux législations et normes administratives et économiques qui codifient les régimes de circulation (limites de poids de bagages pour le transport aérien, normes sanitaires, pression de la demande sur les billets d'avion ou de bateau, fréquence des rotations, etc.). Certains pays sont soumis à un embargo radical, c'est le cas de la Libye, d'autres à des embargos partiels ou des interdictions d'importation ou d'exportation. Sans même évoquer les produits radicalement illicites comme les psychotropes, dont certains voyagent eux aussi en empruntant les routes et les réseaux d'échange que nous décrivons ici, il est assez facile de constater que le passage des différentiels de richesse qui sépare les Nord postindustriels des Sud pauvres, ne sont ni techniquement ni légitimement aisés à franchir. De sorte que ce que nous nommons ici économie de bazar n'est rien d'autre que la somme des arrangements relationnels par lesquels les transferts de marchandises et les transits de personnes sont possibles: le «blanchiment » des douaniers à la frontière, la mobilisation de convoyeurs pour le transport des voitures d'occasion, le transfert de dettes et les investissements différés pour le paiement des marchandises, le groupage du fret, l'obtention de visas, etc. Soit un dispositif dont l'énigme ne tient pas tant à la capacité d'extension et à l'extraordinaire fluidité, ou à la remarquable plasticité, qu'à la constance avec laquelle il maintient la tension ouverte entre local et global et une économie aux dimensions 
d'arrangements relationnels, privilégiant les accords oraux et les rapports de confiance sur les conventions techniques. En effet, si l'échange à ces conditions socio-politiques suppose bien des prouesses individuelles, des ruses et des sommes d'agilité contrebandière dont la grande presse se fait régulièrement l'écho, s'il est avéré également que ces dispositions et dispositifs sont régis par des lois du silence, des dettes d'honneur et parfois de la violence physique, voire des régimes personnels de dépendance, pression, chantage, et autres méthodes criminelles, il est néanmoins impossible de ramener l'ensemble de ces formes d'arrangements, soit à une collection de « prouesses » individuelles, qui n'aurait d'autre sens que de renvoyer à l'ingéniosité des passeurs et à l'urgence de leur situation, pas plus qu'il ne peut se ramener à la rationalité univoque de mafias criminelles organisées. Si évidentes qu'elles paraissent, ces deux représentations par lesquelles on se rend intelligible aujourd'hui dans les sociétés occidentales, cette émergence, occulte le fait que ces arrangements relationnels sont au fond, plus que des prouesses individuelles mais moins que des organisations. Réunir le capital nécessaire à l'ouverture d'une boutique ou au montage d'une affaire; mobiliser autour d'une ou autre activité un réseau de personnes disponibles pour remplacer en cas d'absence, acheminer, livrer, déposer les marchandises sans pour autant être assujetti à des contrats salariaux; être au bon moment et au bon endroit pour se saisir d'une affaire ; faire et défaire aussitôt l'affaire faite des "groupements d'intérêt "; profiter d'un container à demi-vide; telles sont, parmi d'autres, les opérations élémentaires qui mobilisent, ici, comme ailleurs sans doute, les acteurs économiques. Mais elles n'ont pas ici la ressource et la légitimité pour s'appuyer sur des logistiques de circulation des informations et des marchandises, des bases conventionnelles telles que ces opérations puissent se dérouler de façon routinière et mécanique. Si elle sont informelles, elles le doivent moins aux «résistances» et aux ruses qu'elles opposent au contrôle étatique qu'à la double contrainte qu'impose aux acteurs le franchissement de différentiels et de frontières, le rétablissement du cycle marchand interrompu par des filtres politiques et sociaux, sur toute l'étendue des créneaux commerciaux qu'ils occupent. Et ces contraintes ne sont résolues qu'à la condition de bénéficier de trames relationnelles existantes: celle des échanges, dons, contre-dons, influences, informations, pas nécessairement commerciaux, qui parcourent l'étendue des « emplacements » occupés par la dispersion migratoire d'une part, celle des "régions morales " ${ }^{15}$ urbaines favorisant l'échange, la familiarité non pas sur la base d'appartenance commune mais d'une éthique de la performance. Pas de marché ni de rapports marchands sans un dispositif de cafés, bars, restaurants, boîtes de nuit voire tripots, salles de jeux officiels ou clandestins où se nouent des engagements et des réseaux de confiance seulement fondés sur le partage de passions communes et la fréquentation régulière de la passion partagée.

9 Parce qu'il forme un réseau de places marchandes dispersées dans la ville plutôt qu'une enclave, parce que ce «centre se déplace du paroissial et du domestique vers l'importance de l'interjeu entre le local et l'international», parce qu'il permet à différents mondes minoritaires d'être en relations d'affaires, tout en perpétuant les rapports de confiance et d'oralité qui organisent les relations entre "pairs", le dispositif marseillais met en forme ce que V. Ruggiero et N. South nomment « une économie de bazar " ${ }^{16}$. C'est à dire un complexe d'activités articulées où la grande amplitude des circulations commerciales activées par la place marchande, les jeux de franchissement des différentiels, économiques, sociaux, politiques que ces circulations nécessitent, placent ceux qui y font des affaires, entrepreneurs installés, colporteurs, 
consommateurs, en position de "négocier constamment l'acceptabilité morale de leur comportement ${ }^{17}$, dans une situation qui rend possible la coexistence de la légalité et de l'illégalité et le changement permanent des limites entre les deux. Ou le partage du légal et de l'illégal, du moral et de l'immoral, du formel et de l'informel, se fait non pas aux conditions fixées par les normes de la société globale, mais par des négociations en situation dans les scènes publiques ou privées conditionnées par l'échange marchand. De fait, les acteurs, les produits, les réseaux d'échange que nous avons explorés dans les dispositifs commerciaux marseillais relèvent bien d'un cadre informel au sens strictement juridico-politique du terme : ils échappent en effet, dans leur majorité, à toute comptabilité publique et donc au prélèvement fiscal des Etats dont les frontières sont traversées et des pouvoirs locaux dont les territoires sont mobilisés. Il s'est également avéré, comme l'ont démontré des travaux menés sur le commerce de psychotropes ${ }^{18}$ ou les trafics de voitures volées, que les places marchandes où s'opèrent les échanges activés par l'économie de bazar sont aussi des lieux de trafic de produits illicites, au sens légal et moral. D’une façon plus générale, il est démontré que ces produits circulent, parfois sous forme de "fret de retour ", en empruntant les mêmes routes et les mêmes circuits relationnels que les produits informels mobilisés par l'économie de bazar. Qu'il y ait juxtaposition dans l'espace et les temps sociaux de l'échange entre produits licites et illicites, informalité et criminalité, ne suffit pas cependant à confondre ces dynamiques d'échange sous les traits univoques d'organisations souterraines solidaires et "complices " témoignant d'un processus de constitution de mafias. Un tel amalgame, trop souvent pratiqué par des chercheurs comme des gestionnaires urbains, entretient le procès en diabolisation et la désignation stigmatisante dont ces activités commerciales font l'objet. Il est exact que, d'un bord, l'économie de bazar entretient des rapports de contiguïté avec «le capitalisme de pillage $"{ }^{19}$ dont participent les trafics de drogues et qui semble prendre pied dans les quartiers défavorisés des métropoles européennes. Mais il est aussi possible de constater que, d'un autre bord, cette économie entretient des rapports de contiguité avec des « territoires productifs » formels et des activités légales et sédentaires. C'est le cas notamment des districts italiens, fortement impliqués et dynamisés par la fabrication des produits, licites ou informels, mobilisés par l'économie de bazar. Il nous semble de ce point de vue, que c'est l'ensemble de ces rapports qu'il faut analyser, dans une perspective typologique, pour comprendre des modalités de négociation, différenciées et différenciatrices, du couple « nomadisme-sédentarité » et sa place dans les formes contemporaines, urbaines, des économies informelles.

En étendant cette définition à la lumière des travaux que nous avons menés sur Marseille, et que nous poursuivons sur d'autres villes ${ }^{20}$, nous voudrions suggérer ici quelques traits fondamentaux par lesquels l'économie de bazar se distinguerait, dans sa forme aujourd'hui émergente au cœur de nos sociétés postindustrielles, de ce qu'il est convenu d'appeler, de manière par trop générale et uniforme, voire stigmatisante, " économies souterraines " ou "informelles ». Retenons donc, à titre de discussion, au moins trois ensembles de traits distinctifs dont la combinatoire signalerait cette émergence :

- Des dispositions sociales et urbaines qui rendent le partage formel/informel dépendant d'ajustements relationnels en face à face plutôt que de contrats garantis par des institutions ou de règles communautaires. Il ressort des enquêtes que nous avons menées et des propos recueillis auprès de commerçants, que ces accords et les régulations qui en découlent reposent sur une représentation qui fonde les places 
marchandes en "région morale" ${ }^{21}$ : un lieu et des moments dans lesquels les communautés et solidarités de circonstance qui se constituent fonctionnent moins sur le partage d'une ou d'identités culturelles communes (ethniques, religieuses) que sur l'adhésion tacite à une éthique de la performance qui fait de l'engagement dans le commerce quelque chose d'une «aventure» personnelle et d'une «expérience intense » selon le terme de G. Simmel, (1911). De sorte que le commerce y apparaît moins comme moyen d'assurer une survie ou de s'engager dans une carrière, qu'une manière de participer d'une expérience, tout à la fois ascétique et ludique, ayant capacité à "débanaliser la vie quotidienne ", au même titre que certaines aventures individuelles à caractère ludique ou sportif ${ }^{22}$. Dans ces conditions, l'engagement dans les dispositifs commerciaux de «l'économie de bazar» pour des migrants ou des enfants de migrants ne tiendrait pas seulement d'un pis aller faute d'un accès ouvert à la condition salariale, et pas davantage de la perpétuation d'une tradition familiale ou ethnique. L'engagement dans le commerce tiendrait plutôt ici d'une "épreuve " individualisée et individualisante où le risque d'indignité porté par les difficultés de la condition d'étranger est perçue comme défi à relever pour sauver l'honneur. C'est à notre sens ce qui permettrait d'expliquer que l'engagement commercial est singulier (tous les migrants et enfants de migrants ne deviennent pas commerçants ni ne rêvent de le devenir) parce que relevant d'une "expérience extrême" et en même temps relativement banale : car, imaginairement formaté comme compétence performative, il semble ne devoir dépendre que de prouesses individuelles ; l'art de parler et de séduire, la débrouille, la disponibilité et la bonne volonté, le courage, etc.

- Une articulation local/global fondée sur les "compétences à mobilité " des acteurs plutôt que sur des logistiques de transport et des accords transnationaux. Mais cette « compétence à mobilité » n'est pas de fait attachée à la situation de migrant. Elle suppose une condition à la fois singulière et historique qui tient à la plus ou moins grande extension des réseaux migrants, non seulement entre pays d'accueil et pays d'origine, mais plus généralement à l'échelle de vastes territoires capables de constituer des « gisements » de marchandises et de produits disponibles. C'est donc à la condition apparemment paradoxale de superposer une très grande dispersion tout en maintenant la continuité des liens et la facilitation des relations personnelles, que des réseaux migrants peuvent participer efficacement à leur engagement dans l'économie de bazar. Cet engagement serait en somme l'apanage ou la spécificité de groupes portés sur des logiques d'essaimage plutôt que de sédentarisation massive, et donc engagés dans une dynamique diasporique plutôt que migratoire.

- Le franchissement de différentiels et de frontières culturelles, sociales, politiques, comme régime de profitabilité. Ce n'est pas, en soi, la circulation de la marchandise qui détermine les profits, mais la capacité que trouvent les acteurs de leur faire franchir des frontières normalement ou formellement infranchissables. En ce sens, on peut avancer que ce qui ferait la richesse des places marchandes et le caractère stratégique des villes qui les hébergent, tient moins à des conditions géographiques (proximité aux pays d'origine), historiques (tradition marchande), sociales (ancienneté des migrations, main d'œuvre disponible) qu'à la possibilité qu'elles offrent de superposer et de radicaliser de multiples régimes frontaliers, qui sont alors autant d'opportunités commerciales. Même si l'économie de bazar peut être en effet identifiée comme une forme de mobilisation sociale présente dans toutes les métropoles européennes, parce qu'elle est seule aujourd'hui en capacité de mettre sur le marché certains produits, licites ou illicites, c'est dans les villes-frontières posées en des points stratégiques 
balisant les routes commerciales Nord-Sud, qu'elle connaît une véritable expansion et trouve la possibilité d'organiser non pas seulement des activités d'acteurs ponctuels, mais de véritables dispositifs commerciaux tels celui de Belsunce à Marseille.

\section{NOTES}

1. M. Tribalat, Faire France : une enquête sur les immigrés et leurs enfants, Paris, La découverte, 1995.

2. Pour la France voir à ce propos les travaux de S. Pallida, « Le développement des activités indépendantes des immigrés en Europe et en France », in Revue Européenne des Migrations Internationales, vol.8, $n^{\circ} 1,1992$, ainsi que les articles de C.V. Marie, «L'immigration en France dans les années 90 : nouvelle donne pour l'emploi et nouveaux enjeux de société » et E. Ma Mung, «L'entreprenariat ethnique en France », in Sociologie du Travail, vol.XXXVI, n², 1994.

3. Voir notamment les travaux de R. Waldinger, entre autres, Still the promised city? African-american and new immigrants in postindustrial New York, Cambridge, Harvard University Press, 1996 et ceux de S. Sassen, notamment, The global city, Princeton, Princeton University Press, 1991.

4. Sur cette question, voir le travail de E Bonacich et J. Modell, The economic basis of ethnic solidarity, University of california Press, 1980, ou, plus récentes, les différentes études réunies par A. Portes, M. Castells and L. A. Benton, The informal economy ; studies in advanced and less developed countries, Baltimore, The Johns Hopkins University Press, 1991.

5. Tous les chiffres cités ici et plus loin sont tirés du travail pionnier mené par Alain Tarrius sur ce dispositif commercial. Voir notamment, A. Tarrius, L. Missaoui, Arabes de France dans l'économie mondiale souterraine, Marseille, Ed. de l'Aube, 1992.

6. Le terme est emprunté à A. Tarrius, op. cit.

7. Selon l'expression de R. Waldinger (op. cit.) reprise et élargie par J. Rath, « A game of ethnic musical chairs? immigrants and their descendants in the Amsterdam economy ", Communication at the second International Migcities conference, Liège, 6-8 novembre 1997.

8. Confrérie religieuse de rite musulman créée au Sénégal au début du siècle, le mouridisme constitue aujourd'hui une puissance économique, non seulement au Sénégal, mais tout au long des établissements de migrants sénégalais en Afrique, en Europe et depuis plus récemment aux Etats Unis. Combinant prosélytisme religieux et initiatives commerciales de ses membres, valorisant l'entrepreneuriat et le travail auquel le mouridisme donne sens d'engagement religieux, les réseaux mourides constituent aujourd'hui une pièce essentielle de ces économies combinant des formes variées de colportage. Voir à ce propos le travail de V. Ebin, « Les commerçants mourides à Marseille et à New York : regards sur les stratégies d'implantation », in Grégoire E. et Labazée P. (dir.), Grands commerçants d'Afrique de l'Ouest, Paris, Karthala, 1993. Voir également, sur leur implantation en Italie, le travail de O. Schmidt 
di Friedberg, Islam, solidarietà e lavoro ; i muridi senegalesi in Italia, Ed. di la Fondazione Giovanni Agnelli, Torino, 1994.

9. Comme son nom ne l'indique pas, le «marché aux puces» de Marseille n'a que peu de rapport avec les marchés qui portent généralement ce nom. Concédé par la municipalité à une société privée, le marché aux Puces est en réalité une superposition de différents étages commerciaux : marché alimentaire quotidien drainant une partie des « quartiers nord populaires » de la ville, espace public avec ses cafés, restaurants, cabarets, ce marché est aussi un espace commercial ouvert aux chalandises maghrébines (commerce de bazar, confection, électroménager) comme un marché de la brocante. Ses organisateurs ont recensé 30000 visiteurs par week-end en moyenne sur l'année 1993. Voir à ce propos les résultats de nos enquêtes sur ce marché : M. Peraldi, N. Foughali, N. Spinouza, « Le marché des pauvres; espace commercial et espace public », in Revue Européenne des Migrations internationales, vol.11, n¹, 1995. 10. Lorsqu'il était presque exclusivement tourné vers les clientèles algériennes résidentes en Algérie et concentré sur Belsunce, le dispositif commercial recrutait ses commerçants, employés ou simples « fourmis ", plus volontiers en Algérie même ou dans la première génération migrante. Lors d'entretiens, les commerçants exprimaient beaucoup de méfiance vis à vis des « jeunes des cités » réputés paresseux et peu scrupuleux. Avec l'ouverture du marché aux Puces, le ralentissement du commerce vers le Maghreb, l'opportunité offerte par les clientèles locales mais les modifications de style et d'offre qu'elle nécessitait, ont permis l'entrée des « jeunes des cités » dans le monde de l'économie de bazar. Voir à ce propos notre travail sur cette entrée, M. Peraldi, « Portraits d'entrepreneurs », in En marge de la ville, au cœur de la société : ces quartiers dont on parle, (collectif), Marseille, Ed. de l'aube, 1997. C'est l'occasion d'affirmer, une fois encore, que l'appartenance commune à une même origine « ethnique » n'est pas la condition sine qua non, offerte comme un destin « naturel», de la participation aux dispositifs commerciaux, même lorsqu'ils mobilisent exclusivement telle ou telle « communauté ».

11. Certes, les commerçants font plutôt grise mine lorsqu'ils sont restés spécialisés dans les articles de bazar qui avaient les faveurs des clientèles algériennes « là-bas ». Certes encore, le commerce algérien s'est en partie dispersé dans une constellation de places marchandes plus commodes d'accès que Marseille ou sur des frontières plus aisément franchissables : au Maroc (Oujda, Nador ou Casablanca), en Italie (Milan, Gènes ou Naples), en Syrie (Damas), et prenant une place de plus en plus grande dans ce commerce, Istanbul et Dubaï. Marseille reste cependant une place marchande importante, notamment pour le transit des marchandises issues de l'Europe du nord, et principalement les voitures. Le grand marché des voitures d'occasion achetées en Algérie comme celui des pièces auto, reste en grande partie concentré à Marseille et sa région. Selon nos estimations ce sont ainsi près de 1000 véhicules d'occasion qui transitent par Marseille vers l'Algérie chaque mois, vendues par une quinzaine de garages locaux installés à proximité immédiate du quartier « historique » de Belsunce. 12. Voir à ce propos le compte-rendu de nos premières enquêtes dans cette ville, M. Peraldi, « Le cycle algérien dans Lalelli morose », in Bulletin de l'observatoire urbain d'Istanbul, n¹4, juin 1998.

13. Voir à ce propos la très minutieuse description que fait $\mathrm{H}$. Boubakri du commerce transfrontalier entre la Tunisie et la Libye : «Echanges transfrontaliers et commerce parallèle aux frontières tuniso-libyennes, communication au colloque Il Lavoro Informale in Italia e nel Maghreb, Genova, mai 1997. 
14. Cette distinction renvoie aux réflexions de M. Granovetter, « Economic action and social structure : the problem of embeddedness », in American Journal of Sociology, $\mathrm{n}^{\circ} 49,1985$.

15. Selon l'expression de R. Park, La ville, in Y.Grafmeyer et I. Joseph (dir.), L'Ecole de Chicago, naissance de l'écologie urbaine, Paris, Ed. Champ Urbain, 1979. L'expression peut paraître désuète voire déplacée si l'on entend que ces lieux urbains sont affectés d'un indice quelconque de moralité. Chez Park, " moral » s'oppose » à " social », et désigne des lieux qui regroupent des personnes sur leur seule passion commune, hors de tout autre principe de différenciation.

16. V. Ruggiero, N. South, « La ville de la fin de l'ère moderne en tant que bazar : marché des stupéfiants, entreprises illégales et barricades ", in Déviance et société, vol. $20, n^{\circ} 4,1996$. Ces auteurs reprennent ici, en un sens un peu modifié, les termes de C. Geertz, "The Bazaar Economy in Cefrou », in C. Geertz, H. Geertz, L. Rosen (eds.), Meaning and Social Order in Moroccan Society, Cambridge university Press, 1979. C. Geertz y développe une conception de l'économie de bazar que nous ne trahissons pas ici, où cette économie se caractérise d'abord par la forme interpersonnelle, « face to face » des échanges, qui garanti, aux conditions sociales et culturelles qui sont celles du Maroc d'alors, la meilleure efficacité économique possible. Nous ne disons sans doute pas autre chose ici, rapporté aux mondes migrants contemporains. Si étroitement ajustée qu'elle paraisse à une forme urbaine et historique, celle de la ville orientale, trop souvent d'ailleurs ramenée à un trait de mentalité et de culture par les représentations simultanément exotique et stigmatisante qu'elle a généré, l'économie de bazar n'est pas pour autant tributaire et dépendante d'une forme urbaine ou sociale spécifique. Elle nous semble au contraire plutôt nourrie et vivifiée par une double condition relativement intemporelle : l'existence d'une part de filtrages culturels ou politiques et d'adhérences sociales dans le mouvement même de constitution de la marchandise, d'autre part la constitution de régimes différenciés et territorialisés d'altérité dans la ville.

17. V. Ruggiero, N. South, op. cit.

18. Voir à ce propos F. Ingold et alii, Etude sur l'économie souterraine de la drogue : le cas de Paris, IREP, 1995. Ou encore le travail d'A. Tarrius, Héroïne et cocaïne de Barcelone à Perpignan : des économies souterraines ethniques de survie à la généralisation des trafics transfrontaliers de proximité, rapport de recherche OFDT, décembre 1998.

19. Selon l'expression pertinente de L. Wacquant, « de l'Amérique comme utopie à l'envers ", in P. Bourdieu (dir.), La misère du monde, Paris, Seuil, 1993.

20. Les réflexions ici présentées participent d'un programme de recherche soutenu par la Commission Européenne( DG12). Des recherches sont en cours, visant à étendre et affiner les propositions présentées ici, sur Milan, Barcelone, Gènes, Istanbul, Marseille et Anvers.

21. R. Park, La ville, op. cit.

22. A propos de l'éthique sportive partagée on pourra consulter le travail de $\mathrm{L}$. Wacquant, « Protection, discipline et honneur : une salle de boxe dans le ghetto américain ", texte ronéo, juillet 1994. Bon nombre des commerçants que nous rencontrons parlent de leur métier comme d'une passion, une « drogue » disent même certains, qui nécessite « honneur et discipline ». Ajoutons une certaine conception des affaires qui les ramène à des « coups » ou des performances, et l'on comprendra que nous puissions trouver quelques familiarités culturelles entre l'expérience sportive et 
l'expérience commerciale. Ajoutons ce propos d'un dealer récemment interviewé : « je suis comme un footballeur :j'ai commencé le bizness à 18 ans, à 35 ans je raccroche et je m'achète un bar ou un restaurant ».

INDEX

Index géographique : France, méditerranée

Mots-clés : réseaux transnationaux, flux, économie politique, contrebande et trafic, migration 